\title{
Computer-assisted review of digoxin therapy in the elderly
}

\author{
B. WHITING, IRENE WANDLESS ${ }^{1}$, D. J. SUMNER, AND A. GOLDBERG \\ From the Department of Materia Medica and Therapeutics, and the Department of Geriatric Medicine, \\ University of Glasgow, Stobhill General Hospital; and the Department of Clinical Physics and \\ Bio-Engineering, West of Scotland Health Boards, Glasgow
}

Current practice with digoxin was assessed in a group of 42 elderly patients by comparing plasma digoxin concentrations attained on previously established maintenance doses with those generated by a computer programme designed to calculate dosage schedules to suit individual patients. Discrepancies between measured and computed plasma levels and between established and computed doses dictated withdrawal of the drug or revision of dosage in 26 patients (62\%), with obvious clinical benefit. An important determinant of dosage was renal function; reduction in creatinine clearance provided good evidence for the loss of ability of the elderly kidney to eliminate digoxin. Simple bedside methods are available which permit a reliable estimate of creatinine clearance without a 24-hour urine collection, providing a rational basis for the choice of digoxin dosage in the elderly.

Over the past 20 years, a great deal of effort has been directed towards increasing our knowledge of the clinical pharmacology of digoxin and other cardiac glycosides. This has been made possible by the application of isotopic tracer techniques, employing ${ }^{14} \mathrm{C}$ and ${ }^{3} \mathrm{H}$ labelled glycosides, and by the development of assay methods which are capable of measuring small concentrations of digoxin in biological fluids, notably the plasma. Numerous articles on various aspects of the clinical pharmacology of digoxin have been published and most doctors now accept that the therapeutic range of plasma digoxin concentration lies between 1 and $2 \mathrm{ng} / \mathrm{ml}(1.3$ and $2.5 \mathrm{nmol} / \mathrm{l})$ and that knowledge of the plasma concentration may provide a useful guideline in the adjustment of therapy. Whether or not the plasma digoxin concentration is useful in the diagnosis of digoxin toxicity is still a matter of debate (Ingelfinger and Goldman, 1976).

Irrespective of the value of plasma digoxin measurements, much of the work to establish a scientific basis for digoxin therapy has shown the importance of certain characteristics of individual patients; the relation between dose, kinetic behaviour of the drug, and its clinical effect has been

'Present address: Age Research Unit, University of Newcastle upon Tyne, 20 Framlington Place, Newcastle upon Tyne.

Received for publication 4 April 1977 shown to depend upon important factors such as age, sex, body weight, and renal function (Jelliffe, 1967; Kolendorf et al., 1972; Wagner, 1974; Koup et al., 1975; Sumner et al., 1976). These factors have been incorporated into computer programmes designed to calculate drug dosage schedules to suit individual patients (Jelliffe et al., 1972; Peck et al., 1973; Sheiner et al., 1975; Mawer, 1976) and into relatively simple prescribing aids in the form of nomograms (Jelliffe and Brooker, 1974) and formulae (Dobbs et al., 1976; Sumner et al., 1976).

As digoxin therapy in older patients is known to be associated with a number of problems, including the development of toxicity and the question of its long-term usefulness, we decided to investigate the effect of current knowledge of the clinical pharmacology of digoxin on its use in this age group and selected for this purpose a representative group of elderly patients in hospital.

\section{Patients and methods}

A group of 42 elderly patients receiving maintenance digoxin therapy was studied over a three-month period. The patients were drawn from acute assessment wards, rehabilitation units, or long-term care wards in the Glasgow Northern Group of hospitals. In accordance with the policy of this Group, all 
patients were receiving the same brand of digoxin (Lanoxin, Burroughs Wellcome).

After selection of a patient for inclusion in the study, predose ('trough') and 6-hour postdose plasma digoxin concentrations were measured by radioimmunoassay, and a careful record was made of the patient's drug history. Age, sex, weight, a measure of renal function based on at least two serum creatinine estimations, and details of previous digoxin therapy were entered into a computer programme provided by Professor G. E. Mawer of the University of Manchester. This was designed to calculate drug dosage to suit individual patients and to predict corresponding plasma digoxin concentrations at specific times in relation to a dose, viz. predose ('trough') and 6-hours postdose. The programme was of the 'fixed model' type, the rationale of which has been described recently by Mawer (1976). From the age, sex, weight, and serum creatinine, the programme calculated the expected creatinine clearance using equations relating creatinine output to weight and age, with a correction allowing for changing serum creatinine concentration. Assuming a simple one-compartment model and the effective volume of distribution to be a fixed proportion of body weight, the programme then calculated the plasma digoxin concentrations from the drug history, and predicted a digoxin regimen and corresponding plasma levels appropriate to the patient.

The programme was established on file on NUMAC (Northumbrian Multi Access Computer -IBM 360/370) and access was obtained to it from a Teletype Terminal (via a Post Office Modem) in the Department of Materia Medica, Stobhill General Hospital, Glasgow.

Established dosage regimens and measured ('actual') plasma levels were then compared with those generated by the programme, and on the basis of this comparison and the patient's clinical condi- tion at the time a decision whether or not to alter digoxin dosage was made.

As four courses of action were appropriate at this stage, the patients were allocated to one of the following groups: (1) no change in treatment; (2) digoxin stopped; (3) digoxin dose reduced; (4) digoxin dose increased.

The effect of any change in treatment was assessed 3 to 4 weeks later by clinical examination and by further estimations of trough and 6-hour plasma digoxin concentrations. Even when no change was made, digoxin levels were also measured after a 3- to 4-week period.

In many patients who were not incontinent, creatinine clearance was calculated, using either a 6- or a 24-hour urine collection, and this value was compared with corresponding creatinine clearances predicted by the programme.

\section{Results}

Of the 42 patients, 9 were men, aged 70 to 91 years (mean 78 years) and 33 were women, aged 64 to 90 years (mean 77.3 years). All were receiving maintenance digoxin for the treatment of congestive cardiac failure and/or atrial fibrillation. The concomitant use of other drugs was almost universal, and included diuretics, potassium supplements, hypnotics, antibiotics, haematinics, and analgesics. On entry to the study, the daily digoxin dose ('initial dose') ranged from $0.0625 \mathrm{mg}$ to $0.5 \mathrm{mg}$ in four dose categories, viz, $0.0625 \mathrm{mg}, 0.125 \mathrm{mg}$, $0.25 \mathrm{mg}$, and $0.5 \mathrm{mg}$. The commonest daily dose was $0.25 \mathrm{mg}$ ( $43 \%$ of patients) while 29 per cent were taking $0.125 \mathrm{mg}$ daily and 26 per cent $0.0625 \mathrm{mg}$ daily; only one patient was taking $0.5 \mathrm{mg}$ daily.

On the basis of both the comparison between actual and predicted data and clinical examination on entry to the study, the patients were divided into the 4 groups shown in the Table.

Table Details of 4 groups of patients studied

\begin{tabular}{|c|c|c|c|c|}
\hline & $\begin{array}{l}\text { Group } 1 \\
\text { No change }\end{array}$ & $\begin{array}{l}\text { Group } 2 \\
\text { Digoxin stopped }\end{array}$ & $\begin{array}{l}\text { Group } 3 \\
\text { Dose reduced }\end{array}$ & $\begin{array}{l}\text { Group } 4 \\
\text { Dose increased }\end{array}$ \\
\hline 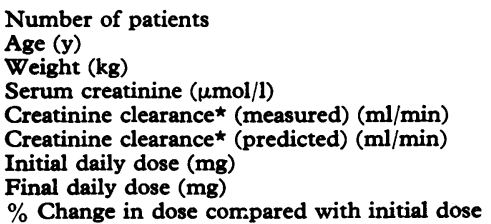 & $\begin{aligned} & 16 \\
& 73 \cdot 25 \pm 6 \cdot 1 \\
& 53 \cdot 25 \pm 9 \cdot 3 \\
& 108 \cdot 7 \pm 27 \cdot 7 \\
& 44 \cdot 2 \pm 18 \cdot 3 \\
& 41 \cdot 6 \pm 17 \cdot 4 \\
& 0 \cdot 211 \pm 0 \cdot 060 \\
& 0 \cdot 211 \pm 0 \cdot 060 \\
&-\end{aligned}$ & $\begin{array}{l}6 \\
81 \cdot 2 \pm 4 \cdot 5 \\
59 \cdot 6 \pm 9 \cdot 3 \\
113 \cdot 8 \pm 14 \cdot 9 \\
36,26,55 \\
37 \cdot 3 \pm 11 \cdot 6 \\
0 \cdot 083 \pm 0 \cdot 032 \\
\text { Nil } \\
-\end{array}$ & $\begin{aligned} 10 & \\
78 \cdot 5 & \pm 5 \cdot 3 \\
48 \cdot 8 & \pm 10 \cdot 5 \\
117 \cdot 4 & \pm 31 \cdot 9 \\
52,22 & , 52,19 \\
30 \cdot 5 & \pm 8 \cdot 8 \\
0 \cdot 264 & \pm 0 \cdot 098 \\
0 \cdot 161 & \pm 0 \cdot 035 \ddagger \\
35 \cdot 7 & \pm 14 \cdot 4\end{aligned}$ & $\begin{aligned} 10 & \\
80 \cdot 4 & \pm 6 \cdot 7 \\
48 \cdot 3 & \pm 9 \cdot 1 \\
118 \cdot 4 & \pm 29 \cdot 6 \\
35,24 & , 37 \\
29 \cdot 5 & \pm 9 \cdot 0 \\
0 \cdot 081 & \pm 0 \cdot 030 \dagger \\
0 \cdot 153 & \pm 0 \cdot 042 \ddagger \\
103 \cdot 6 & \pm 79 \cdot 2\end{aligned}$ \\
\hline
\end{tabular}

Results are expressed as mean \pm SD.

* No significant difference between measured and predicted creatinine clearance in 21 patients (paired t test).

†Difference in initial dose between groups 3 and 4 is significant. $P<0.001$ ( $t$ test).

$¥$ Difference in final dose between groups 3 and 4 is not statistically significant. 
GROUP 1

Sixteen patients (38\%) required no change in digoxin dosage, which was satisfactory and conformed to that recommended by the programme (Fig. 1). Actual trough and 6-hour plasma digoxin concentrations were spread throughout the subtherapeutic and therapeutic range and were similar to but slightly less than those predicted on the basis of the drug history (Fig. 2). The clinical condition of this group of patients was satisfactory. With no change in prescribed treatment, a significant rise in both trough and 6-hour levels $(P<0.001)$ was seen when plasma digoxin concentrations were remeasured after a period of 3 to 4 weeks (Fig. 3), and at this time, though there were some discrepancies, actual trough and 6-hour levels were similar to those predicted (Fig. 2).

GROUP 2

Six patients (14\%) had initial plasma digoxin concentrations well below the therapeutic range associated with predicted concentrations which were also subtherapeutic (Fig. 2). Doses in this group were correspondingly low (mean $0.083 \mathrm{mg}$
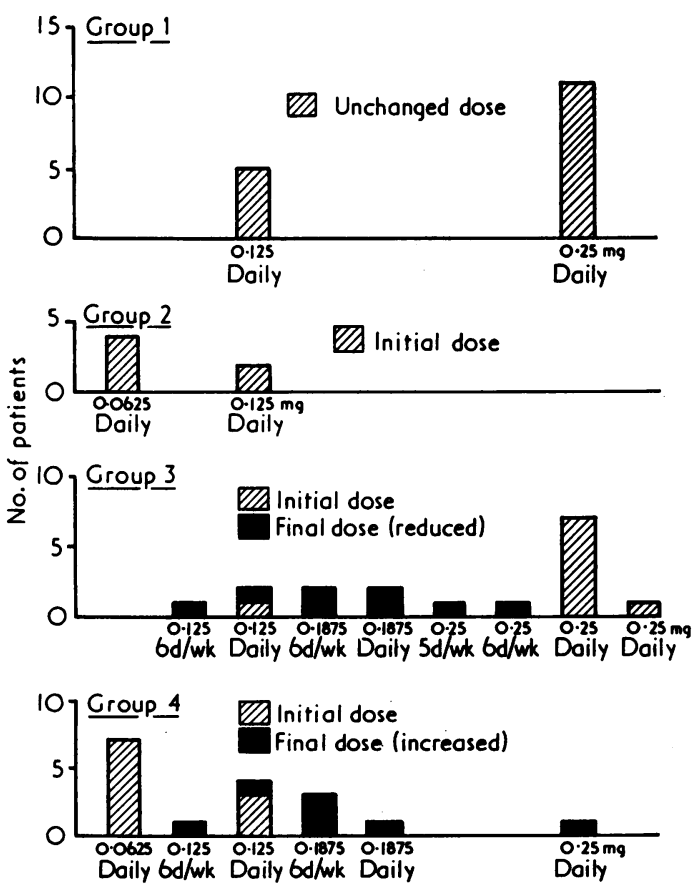

Fig. 1 Histograms of the initial (entry) and final (computed) digoxin doses in the 4 groups involved in the study : group 1, no change in dosage; group 2, digoxin stopped; group 3, dose reduced; group 4, dose increased. daily) (Fig. 1) but the absence of any signs of congestive cardiac failure or tachyarrhythmia suggested that there was no need for further digoxin therapy. This impression was confirmed when the clinical condition of the patients was found to be stable for at least 4 weeks after stopping digoxin. Plasma digoxin concentrations corresponding to doses that would be appropriate for patients in this group are also shown in Fig. 2.

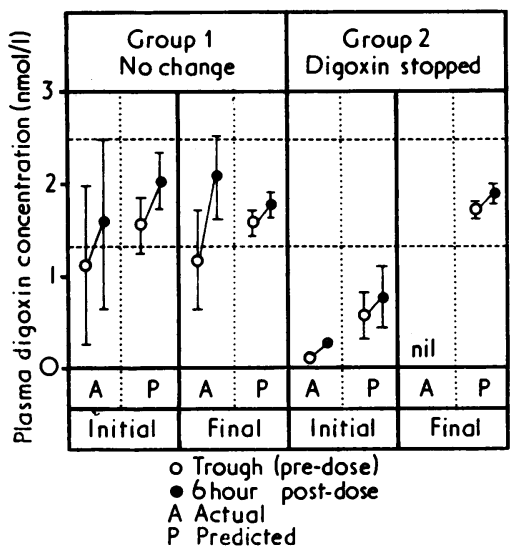

Fig. 2 Initial and final measured plasma digoxin concentrations ('actual') and levels predicted by the computer programme ('predicted') in patients who required no change in dosage (group 1) or in whom the drug was stopped (group 2). Vertical bars represent mean $\doteq S D$.

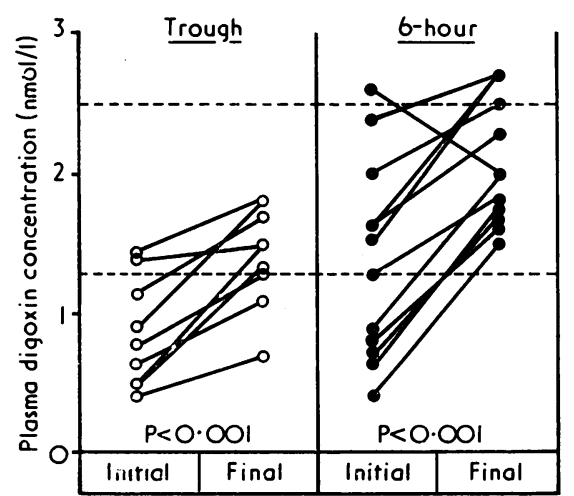

Fig. 3 Comparison of trough and 6-hour plasma digoxin concentrations at the beginning ('initial') and at the end ('final') of the study, in patients not requiring any change in dosage (group 1) and in whom estimations were repeated at 3 to 4 weeks. $P$ values are based on the paired $t$ test. 


\section{GROUP 3}

Ten patients $(24 \%)$ had high initial plasma digoxin concentrations (Fig. 4) which were associated with inappropriately high doses (mean $0.264 \mathrm{mg}$ daily) when compared with those recommended by the programme (mean $0 \cdot 161 \mathrm{mg}$ daily). Fig. 1 shows the initial doses and those recommended by the programme, which were subsequently adopted. The effect of this reduction in dosage on plasma digoxin concentrations can be seen in Fig. 4, which also shows the good agreement between actual and predicted levels. The therapeutic range had obviously been achieved when plasma digoxin concentrations were measured again 3 to 4 weeks after the change in dosage.

Seven patients in this group had typical extracardiac symptoms of digoxin toxicity including anorexia, nausea, and vomiting. These symptoms subsided completely on reduction of dosage. One patient with a trough level of $3 \mathrm{nmol} / \mathrm{l}$ and a 6 -hour level of $3.9 \mathrm{nmol} / 1$ also had evidence of cardiac toxicity in the form of multifocal ventricular and supraventricular extrasystoles; after reduction in digoxin dosage, the supraventricular extrasystoles were abolished and the frequency of ventricular extrasystoles considerably reduced. The other 3 patients in this group showed no signs of digoxin toxicity but had high plasma digoxin concentrations and doses in excess of those recommended by the programme.

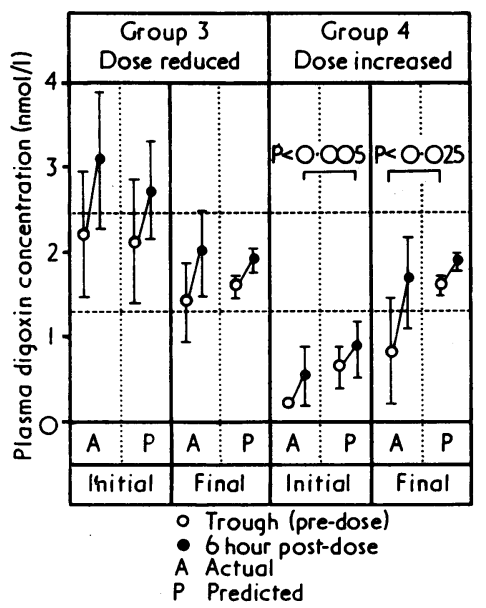

Fig. 4 Initial and final measured plasma digoxin concentrations ('actual') and levels predicted by the computer programme ('predicted') in patients who required a reduction (group 3) or an increase (group 4) in dosage. Vertical bars represent mean $\pm S D$. $P$ values are based on the paired $t$ test.

\section{GROUP 4}

Ten patients $(24 \%)$ had low initial plasma digoxin concentrations (Fig. 4) which were associated with inappropriately low doses (mean $0.081 \mathrm{mg}$ daily) when compared with those recommended by the programme (mean $0.153 \mathrm{mg}$ daily). Fig. 1 shows the initial doses and those recommended by the programme, which were subsequently adopted. The effect of this increase in dosage on plasma digoxin concentrations can be seen in Fig. 4. Whereas a significant difference existed between actual and predicted 6-hour levels initially $(P<0.005)$, there was good agreement between actual and predicted levels after increase in dosage, and in most patients a therapeutic level was achieved. Actual trough levels at this time, however, were significantly less than those predicted $(P<0.025)$.

From a clinical standpoint, frank congestive cardiac failure was controlled by the increase in dosage in two patients in this group, while there was an average 19 per cent reduction in the ventricular rate in another 7 patients with inadequately controlled atrial fibrillation. The clinical condition of the remaining patient was initially satisfactory and remained so with the increased dosage.

Measured and predicted creatinine clearance values in 21 continent patients of all groups compared very favourable. There was no statistically significant difference between measured values $(39 \cdot 4 \pm 16 \cdot 2$ (SD) $\mathrm{ml} / \mathrm{min}$ ) and predicted values (36.2 \pm 14.0 (SD) $\mathrm{ml} / \mathrm{min}$ ) (paired t test). Reference to the Table shows that there was no significant difference between predicted creatinine clearance values in groups 3 and 4 , and that these groups were also similar with respect to age and weight. Initial digoxin doses in these groups differed significantly (group 3, $0.264 \pm 0.098$ (SD) $\mathrm{mg}$ daily; group 4, $0.081 \pm 0.030$ (SD) $\mathrm{mg}$ daily; $\mathrm{P}<0.001$ ) but final computed doses were similar (group 3, $0.161 \pm$ 0.035 (SD) $\mathrm{mg}$ daily; group 4, $0.153 \pm 0.042$ (SD) $\mathrm{mg}$ daily). The Table also shows that the average digoxin dose was reduced by approximately onethird in group 3 but was doubled in group 4 .

Only one patient, who showed no signs of digoxin toxicity, had a subnormal potassium level ( 3.3 $\mathrm{mmol} / \mathrm{l}$, normal range 3.5 to $5.5 \mathrm{mmol} / \mathrm{l}$ ) initially. On the basis of clinical examination and measurement of $T_{3}$ and $T_{4}$ levels, all patients were euthyroid.

\section{Discussion}

This study was designed to assess the effect of recent advances in the clinical pharmacology of digoxin on the use of this drug in clinical practice. A group of elderly patients was chosen because of 
the particular problems encountered in this age group, notably toxicity. Many studies have highlighted the dangers of digoxin therapy in the elderly and Dall (1970) has shown that the drug can be stopped without detriment in a large proportion of elderly patients. Digoxin, therefore, has a certain 'nuisance value' which is related to its narrow therapeutic ratio and to doubts about its long-term effectiveness. As it is eliminated primarily by glomerular filtration, renal function is an important determinant of dosage and the importance of this in the treatment of elderly patients was shown quite clearly in the present study by the low creatinine clearance values, obtained both by direct measurement and by computer prediction. A mean (predicted) value of $35.6 \pm 13.7$ (SD) $\mathrm{ml} / \mathrm{min}$ for the whole group highlighted the inevitable deterioration in renal function which is associated with advancing age (Watkin and Shock, 1955) and which is explainable on the basis of Bricker's 'intact nephron hypothesis' (Bricker et al., 1960). The adjustments in dose required in groups 3 and 4 were related principally to renal function in these groups, and the slightly higher creatinine clearance values in group 1 accounted for correspondingly higher doses. As the creatinine clearance is the individual patient characteristic which relates most closely to digoxin dosage requirement (Dobbs et al., 1976), its insertion into the formula

Maintenance dose $=$

$$
\frac{0.0025 \text { (creatinine clearance }+47 \text { ) }}{\text { fraction absorbed }}{ }^{\star} \mathrm{mg} / \text { day }
$$

as described previously by Sumner et al. (1976) can predict doses appropriate for reduced creatinine clearance rates:

$\begin{array}{cl}\text { Creatinine clearance } & \text { Daily dose of digoxin } \\ 8-25 \mathrm{ml} / \mathrm{min} & 0 \cdot 125 \mathrm{mg} \\ 26-49 \mathrm{ml} / \mathrm{min} & 0 \cdot 1875 \mathrm{mg} \\ 50-79 \mathrm{ml} / \mathrm{min} & 0 \cdot 25 \mathrm{mg}\end{array}$

A good estimate of the creatinine clearance in an individual patient can be obtained from the age, sex, body weight, and steady serum creatinine concentration by use of the nomogram designed by Kampmann et al. (1974). Application of this nomogram and the formula shown above gives dosage recommendations which are broadly consistent with those derived from the computer programme.

Approximately two-thirds of the patients required some form of intervention as a result of this study. This implies that only about 1 patient in 3 was receiving the correct dose when measured against computed doses. The subsequent clinical course of many of the patients confirmed the validity

*The fraction absorbed depends on the bioavailability of the particular digoxin preparation. of this approach. Toxic manifestations were relieved and improved control of cardiac failure and ventricular rate was achieved. The drug was stopped in a small number of patients $(14 \%)$ when initial observations suggested that inappropriately small doses associated with subtherapeutic plasma digoxin concentrations could not be exerting any useful effect. The absence of cardiac failure in these patients before and after withdrawal of digoxin confirmed this view. No attempt was made to assess the ultimate requirement for digoxin in all patients, but it is probable that within the group requiring no change in treatment a similar policy of withdrawing the drug could have been adopted. Even so, the number of patients not requiring long-term therapy would not have reached the figure of approximately 75 per cent reported by Dall (1970). Perhaps a more positive attitude is required towards withdrawing digoxin when there is doubt about the need for the drug in individual patients.

The dosage recommendations made by the programme made it obvious that a considerable number of patients were receiving doses inconsistent with their age, sex, body weight, and renal function. Patients in group 3 were receiving an inappropriately high dose while patients in groups 2 and 4 were receiving an inadequate dose. The reasons for this are probably complex but may include alterations in renal function with advancing age or intercurrent disease which would effectively reduce the elimination of digoxin. On the other hand, an overconservative attitude towards the prescribing of digoxin would impose inappropriate limitations on dosage; indeed, a twofold increase in dosage was required to achieve satisfactory control of cardiac failure or to improve the control of atrial fibrillation in patients who were inadequately dosed. It is interesting to note that the traditional 'geriatric' daily dose of $0.0625 \mathrm{mg}$ was employed in 11 patients $(26 \%)$ on entry to the study, but that subsequently no patient received such a small dose. The computer programme permitted a degree of control over dosage which is not normally practised, but which is feasible by simple manipulation of standard digoxin preparations as shown in Fig. 1.

Throughout the four groups, there was relatively good agreement between actual and predicted levels, but this was subject to some interesting variation. Slight discrepancies may have been the result of an inability of the programme to recognise variations in digoxin bioavailability from one patient to another; a relatively uniform degree of digoxin absorption was assumed, viz. 60 per cent for 'Lanoxin' tablets (Huffman et al., 1974; Johnson and Bye, 1975), which is not entirely realistic in practice. Individual differences in absorption, dis- 
tribution, and extrarenal elimination may also have accounted for the relatively large standard deviations associated with measured plasma digoxin concentrations. The discrepancies also reflected another important source of variation which was most noticeable in those patients not requiring any change in therapy. Fig. 3 shows that actual (measured) levels of plasma digoxin rose significantly without any apparent change in dosage over the period covered by the study, and there is no doubt that improved compliance contributed to this, even within a hospital setting. A recent study (Wandless and Davie, 1977) in a similar group of elderly patients in hospital has shown that compliance is by no means perfect. The present kind of study can, however, increase the interest of both patients and nursing staff in control of digoxin therapy to effect some improvement in compliance.

There seems no doubt that an individualised approach to digoxin therapy is desirable and that benefit can only result from a review of existing treatment in individual patients. Such a review can be facilitated by the measurement of plasma digoxin concentration and by revision of dosage regimens in the light of individual patient characteristics, notably renal function. Computational facilities need not be available, but use of simple prescribing aids, such as the formula illustrated above which relates the maintenance dose to the desired plasma concentration and total digoxin clearance, would help to prevent many of the errors which occur as a result of intuitive prescribing.

We are grateful to the physicians who allowed us to study patients under their care, to Dr. Wendy Ratcliffe for carrying out plasma digoxin estimations by radioimmunoassay, and to Professor G. E. Mawer for helpful discussion and for making the drug dosage programme available. A grant from the Greater Glasgow Health Board (Higher Medicine Fund) for the purchase of the computer terminal is also gratefully acknowledged.

\section{References}

Bricker, N. S., Morrin, P. A. F., and Kime, S. W. (1960).

The pathologic physiology of chronic Bright's disease. American fournal of Medicine, 28, 77-98.

Dall, J. L. C. (1970). Maintenance digoxin in elderly patients. British Medical fournal, 2, 705-707.

Dobbs, S. M., Mawer, G. E., Rodgers, E. M., Woodcock,
B. G., and Lucas, S. B. (1976). Can digoxin dose requirements be predicted? British fournal of Clinical Pharmacology, 3, 231-237.

Huffman, D. H., Manion, C. V., and Azarnoff, D. L. (1974). Absorption of digoxin from different oral preparations in normal subjects during steady state. Clinical Pharmacology and Therapeutics, 16, 310-317.

Ingelfinger, J. A., and Goldman, P. (1976). The serum digitalis concentration-does it diagnose digitalis toxicity? New England fournal of Medicine, 294, 867.

Jelliffe, R. W. (1967). A mathematical analysis of digitalis kinetics in patients with normal and reduced renal function. Mathematical Biosciences, 1, 305-325.

Jelliffe, R. W., and Brooker, G. (1974). A nomogram for digoxin therapy. American fournal of Medicine, 57, 63-68.

Jelliffe, R. W., Buell, J., and Kalaba, R. (1972). Reduction of digitalis toxicity by computer-assisted glycoside dosage regimens. Annals of Internal Medicine, 77, 891-906.

Johnson, B. F., and Bye, C. (1975). Maximal intestinal absorption of digoxin, and its relation to steady state plasma concentration. British Heart Fournal, 37, 203-208.

Kampmann, J., Siersbaek-Nielsen, K., Kristensen, M., and Mølholm Hansen, J. (1974). Rapid evaluation of creatinine clearance. Acta Medica Scandinavica, 196, 517-520.

Kolendorf, K., Christiansen, N. J. B., Siersbaek-Nielsen, K., and Mølholm Hansen, J. (1972). Serum digoxin after dosage regimen based on body weight and renal function (letter). Lancet, 2, 326.

Koup, J. R., Jusko, W. J., Elwood, C. M., and Kohli, R. K. (1975). Digoxin pharmacokinetics: role of renal failure in dosage regimen design. Clinical Pharmacology and Therapeutics, 18, 9-21.

Mawer, G. E. (1976). Computer assisted prescribing of drugs. Clinical Pharmacokinetics, 1, 67-78.

Peck, C. C., Sheiner, L. B., Martin, C. M., Combs, D. T., and Melmon, K. L. (1973). Computer-assisted digoxin therapy. New England fournal of Medicine, 289, 441-446.

Sheiner, L. B., Halkin, H., Peck, C. C., Rosenberg, B., and Melmon, K. L. (1975). Improved computer-assisted digoxin therapy. A method using feed-back of measured serum digoxin concentrations. Annals of Internal Medicine, 82, 619-627.

Sumner, D. J., Russell, A. J., and Whiting, B. (1976). Digoxin pharmacokinetics: multicompartmental analysis and its clinical implications. British fournal of Clinical Pharmacology, 3, 221-229.

Wagner, J. G. (1974). Loading and maintenance doses of digoxin in patients with normal renal function and those with severely impaired renal function. Fournal of Clinical Pharmacology, 14, 329-338.

Wandless, I., and Davie, J. W. (1977). Can drug compliance in the elderly be improved? British Medical fournal, 1, 359-361.

Watkin, D. M., and Shock, N. W. (1955). Agewise standard value for $\mathrm{C}_{\mathrm{IN}}, \mathrm{C}_{\mathrm{PAH}}$ and $\mathrm{Tm}_{\mathrm{PAH}}$ in adult males (abstract). fournal of Clinical Investigation, 34, 969.

Requests for reprints to Dr. Brian Whiting, Department of Materia Medica, Stobhill General Hospital, Glasgow G21 3UW. 